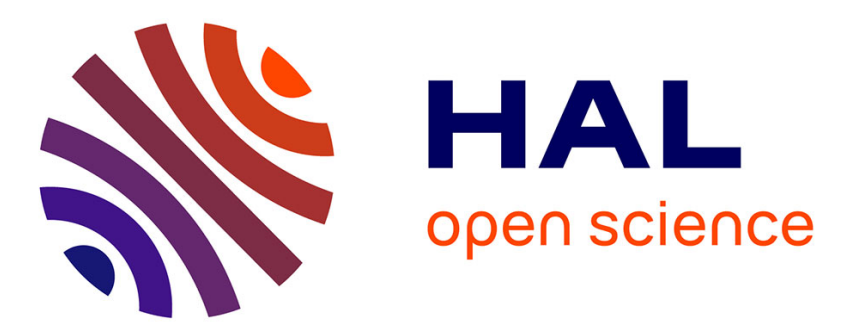

\title{
A Linear Time-Varying Controller for Synchronization of LÜ Chaotic Systems With One Input
}

Antonio Loria

\section{To cite this version:}

Antonio Loria. A Linear Time-Varying Controller for Synchronization of LÜ Chaotic Systems With One Input. IEEE Transactions on Circuits and Systems I: Regular Papers, 2009, 56 (08), pp.674 678. 10.1109/TCSII.2009.2025624 . hal-00447345

\section{HAL Id: hal-00447345 \\ https://hal.science/hal-00447345}

Submitted on 14 Jan 2010

HAL is a multi-disciplinary open access archive for the deposit and dissemination of scientific research documents, whether they are published or not. The documents may come from teaching and research institutions in France or abroad, or from public or private research centers.
L'archive ouverte pluridisciplinaire HAL, est destinée au dépôt et à la diffusion de documents scientifiques de niveau recherche, publiés ou non, émanant des établissements d'enseignement et de recherche français ou étrangers, des laboratoires publics ou privés. 


\title{
A Linear Time-Varying Controller for Synchronization of Lü Chaotic Systems With One Input
}

\author{
Antonio Loría
}

\begin{abstract}
This brief addresses the problem of synchronization of two Lü chaotic systems. As opposed to nonlinear Lyapunov-based controllers, which are designed with the aim of rendering the derivative of a Lyapunov function negative along trajectories of the closed-loop system, control design exploits the natural stability properties of the system. The proof relies not only on basic Lyapunov stability theory but also on other concepts, such as persistency of excitation. The contribution with respect to other works is to show that one control input is enough to force synchronization; moreover, the controllers are linear time varying.
\end{abstract}

Index Terms-Chaotic systems, control, nonlinear oscillators, synchronization.

\section{INTRODUCTION}

$\mathbf{M}$ ASTER-SLAVE synchronization continues to attract the attention of research communities. For example, the utility of popular techniques such as observer-based synchronization (cf. [2] and [5]) is reiterated by experiment validation as feasible solutions for secure telecommunication (cf. [4]). In that context, see also [6] for a brief paper on the synchronization of Lü systems (which are the subject of this brief) in the context of code-division multiple access.

This brief addresses the problem of controlled master-slave synchronization for the so-called Lü chaotic oscillator (cf. [9]); this problem was successively solved via nonlinear Lyapunovbased control in [10] and [11], in the former using three control inputs and in the latter using two control inputs. In both references, control is nonlinear and designed with the aim of rendering the derivative of a quadratic Lyapunov function negative definite, which is a standard procedure in control theory.

Our controllers are designed, following engineering intuition (basic "filtering signal" ideas) and a fundamental concept from stability and control theory named persistency of excitation (cf. [1]). Roughly, the latter is the property of a signal by which a certain average is strictly positive. For illustration, consider the context of electrical circuits, particularly a standard (serial) $R C$ circuit $^{1}$ with variable resistance $R(t)$, which may be zero for "large" periods of time. During such intervals, it is clear that passive element $R$ becomes lossless, i.e., energy dissipation is lost. At intervals over which $R=0$ becomes larger, energy

Manuscript received November 16, 2008; revised March 17, 2009. First published July 14, 2009; current version published August 14, 2009. This paper was recommended by Associate Editor B. Jean-Pierre.

The author is with the Centre National de la Recherche Scientifique, Laboratoire des Signaux et Systèmes, 91192 Gif sur Yvette, France (e-mail: loria@1ss.supelec.fr).

Color versions of one or more of the figures in this paper are available online at http://ieeexplore.ieee.org.

Digital Object Identifier 10.1109/TCSII.2009.2025624

${ }^{1}$ With the current through the resistor as input and the capacitor voltage as output. is stored "longer" in the capacitor element. For energy to be dissipated (on average), it seems reasonable to impose a maximum interval length over which $R \approx 0$ or, in other words, that $R(t)$ has a strictly positive minimum "average" value. This idea, which is formally captured in the property of persistency of excitation, is our main steering intuition to show that the controlled synchronization of two Lü chaotic oscillators may be achieved with one linear time-varying control input. This is our main contribution with respect to existing literature.

The remainder of this brief consists of the main results (cf. Section II), and stability proofs are given in Section III.

\section{MAIN RESUltS}

\section{A. Problem Formulation}

The Lü chaotic system is given by (cf. [9])

$$
\begin{aligned}
& \dot{x}_{m}=\beta x_{m}-y_{m} z_{m}+c \\
& \dot{y}_{m}=-a y_{m}+x_{m} z_{m} \\
& \dot{z}_{m}=-b z_{m}+x_{m} y_{m} .
\end{aligned}
$$

Unless otherwise specified, it is conventionally assumed that the constant parameters $\beta, a$, and $b$ are strictly positive and that $c \in \mathbb{R}$. We assume that system (1) freely "moves" about, so it is called the master system. The synchronization problem is to design a controller $\left(u_{1}, u_{2}, u_{3}\right)$ such that the trajectories of the slave system

$$
\begin{aligned}
& \dot{x}_{s}=\beta x_{s}-y_{s} z_{s}+c+u_{1} \\
& \dot{y}_{s}=-a y_{s}+x_{s} z_{s}+u_{2} \\
& \dot{z}_{s}=-b z_{s}+x_{s} y_{s}+u_{3}
\end{aligned}
$$

asymptotically follow those of the master, i.e., it is desired that

$$
\begin{aligned}
& \lim _{t \rightarrow \infty}\left|x_{s}-x_{m}\right|=0 \\
& \lim _{t \rightarrow \infty}\left|y_{s}-y_{m}\right|=0 \\
& \lim _{t \rightarrow \infty}\left|z_{s}-z_{m}\right|=0 .
\end{aligned}
$$

This problem was solved via Lyapunov-based nonlinear control in [10] and [11]. In the latter, it is shown that two control inputs are enough to produce synchronization: In particular, the authors propose two controllers that exponentially synchronize the systems with either $u_{2}=0$ or $u_{3}=0$. Control input $u_{1}$ is used in both references to compensate for the term $\beta x_{s}$, $\beta>0$, which induces instability. In the case where $y_{s}$ and $z_{s}$ are different from zero, a term of undefined sign is added to (2a); hence, the latter may be regarded as an unstable system with a nonvanishing disturbance (cf. [7]), which is expected to be unstable. Both [10] and [11] rely on canceling unstable terms; however, canceling unstable "poles" is, in general, undesirable due to parametric uncertainty. In this brief, it is shown that 
the systems may be synchronized with $u_{1}=0$ for appropriate choices of $u_{2}$ or $u_{3}$. That is, it is shown that synchronization can be achieved with one control input: hence, either $u_{1}=$ $u_{2}=0$ or $u_{1}=u_{3}=0$. Moreover, the controller is designed by exploiting and not dismantling the system's natural structure.

To put the main results into perspective, the synchronization error dynamics is first written. To that end, define

$$
e_{1}:=x_{s}-x_{m} \quad e_{2}:=y_{s}-y_{m} \quad e_{3}=z_{s}-z_{m} .
$$

Then, subtracting (1) from (2), we obtain

$$
\begin{aligned}
& \dot{e}_{1}=\beta e_{1}-y_{s} e_{3}-z_{m} e_{2}+u_{1} \\
& \dot{e}_{2}=-a e_{2}+z_{m} e_{1}+x_{s} e_{3}+u_{2} \\
& \dot{e}_{3}=-b e_{3}+x_{s} e_{2}+e_{1} y_{m}+u_{3} .
\end{aligned}
$$

The synchronization problem is solved if and only if it is ensured that $\lim _{t \rightarrow 0} e_{i}(t)=0$ for all $i$. Beyond a mere convergence property, one may seek the stronger property of asymptotic stability of $e=0 .^{2}$ A yet stronger property is exponential stability, which is proven in [10] and [11] using Lyapunov's direct method and at least two control inputs including $u_{1}$.

\section{B. Solution}

Set $u_{1} \equiv 0$. We start by posing some evidently abusive assumptions with the sole purpose of illustrating the idea of our control approach; formal statements are made later.

Step 1) In order to circumvent the inherent instability of (5a) with $u_{1} \equiv 0$, either $e_{2}$ or $e_{3}$ is regarded as a virtual control input. For instance, with $e_{3}$ being a control input (the same reasoning is valid for $e_{2}$ ), let $e_{3}:=$ $k_{2} y_{s} e_{1}$, where $k_{2}$ is a control gain, and let $e_{2}=0$. Then, (5a) reads

$$
\dot{e}_{1}=\beta e_{1}-k_{2} y_{s}(t)^{2} e_{1} .
$$

The term $-k_{2} y_{s}^{2}$ is nonpositive; hence, $-k_{2} y_{s}^{2} e_{1}$ is considered to be a stabilizing term used to compensate for the destabilizing term $\beta e_{1}$ with $\beta>0$. For instance, it is clear that if $y_{s}$ is constant, it suffices to pick $k_{2}>\beta / y_{s}^{2}$ to make the origin $e_{1}=0$ of (6) exponentially stable. Yet, in the context of chaotic systems, it certainly does not make sense to assume that $y_{s}$ is constant; moreover, most typically, $y_{s}(t)=0$ for certain values of $t$ since it corresponds to the trajectory of the slave system. For those instances, infinite gain is seemingly needed. However, it reasonably may be assumed that the set of times $t$ such that $y_{s}(t)=0$ are isolated points or, at least, that $y_{s}(t) \neq 0$ for intervals of time of a minimal length. Then, it is said that $y_{s}$ has the property of persistency of excitation, which is comparable to an "average value" and is mathematically written as follows (cf. [1] and [8]):

Definition 1: The locally integrable function $\varphi: \mathbb{R}_{\geq 0} \rightarrow \mathbb{R}$ is said to be persistently exciting (PE) if there exist positive numbers $\mu$ and $\Delta$ such that

$$
\int_{t}^{t+\Delta} \varphi(s)^{2} d s \geq \mu>0 \quad \forall t \geq 0 .
$$

\footnotetext{
${ }^{2}$ Convergence and asymptotic stability are different properties that are sometimes mistakenly taken for synonyms (cf. [7]).
}
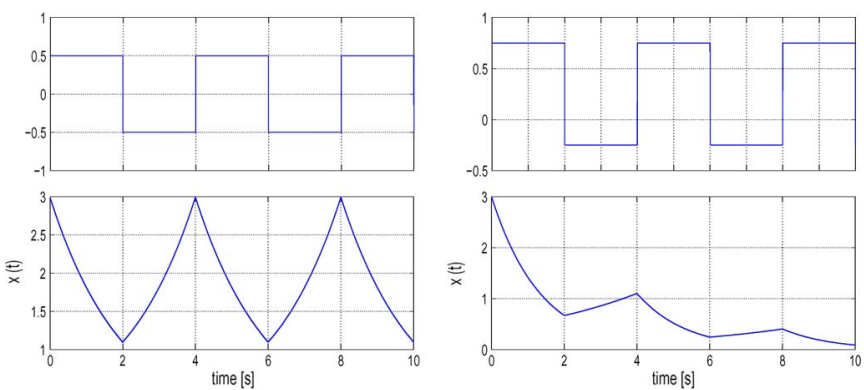

Fig. 1. (a) Graph of (top) $\varphi(t)^{2}-0.5$ and (bottom) corresponding $x(t)$. (b) Graph of (top) $\varphi(t)^{2}-0.25$ and (bottom) corresponding $x(t)$.

It has been well known for many years (cf. [1]) that the origin of $\dot{x}=-\varphi(t)^{2} x$ is exponentially stable if and only if $\varphi(t)$ is PE. ${ }^{3}$ The rationale of this statement may be understood with the following example: Let $\varphi(t)=0$ for all $t \in(2 i, 2 i+2]$, with $i>0$ being odd, and $\varphi(t)=1$ for all $t \in(2 i, 2 i+2]$, with $i$ being even. Then, $\dot{x}(t)=-x(t)$ on intervals where $i$ is even and $x(t)$ exponentially decays, whereas $x(t)=$ const on intervals for odd values of $i$. See that $x(t)$ exponentially decays as $t \rightarrow \infty$. Consider now the system $\dot{x}=-\left[\varphi(t)^{2}-\beta\right] x$ with $\beta>0$. Provided that $\beta<1$, over the intervals where $\varphi(t)^{2}>\beta$, solution $x(t)$ decays exponentially fast with rate $1-\beta$; during the complementary intervals, $|x(t)|$ exponentially increases with a rate equal to $\beta$. Graphically (cf. Fig. 1), one can see that the system's trajectories exponentially decay to zero if $\beta<0.5$.

The previous example may be put in the context of the $R C$ circuit previously evoked by considering $\varphi(t)^{2}$ as the value of the variable resistance and $\beta$ as a constant supply that keeps the circuit from dissipating energy, so that the capacitor does not discharge. Note that, for simplicity, the "operating point" is zero, but this is assumed without loss of generality. We formalize the previous reasoning in the following technical lemma:

Lemma 1: Consider the system

$$
\dot{x}=-\varphi(t) x, \quad \varphi(t):=[a(t)-b], \quad x \in \mathbb{R}
$$

where $a(t) \geq 0$ is globally Lipschitz and bounded; $b>0$; and there exist positive numbers $\mu_{m}, \mu_{M}$, and $\Delta$ such that

$$
\mu_{m} \geq \int_{t}^{t+\Delta} \varphi(s) d s \geq \mu_{m}>0 \quad \forall t \geq 0 .
$$

Then, the origin of (8) is exponentially stable for suitable values of $\mu_{m}, \Delta$, and $b$.

The proof is included in Section III. Roughly, as it may be inferred from the previous discussion and Fig. 1, it is required that $\mu_{m}$ and $\Delta$ be sufficiently large; this implicitly requires that $a(t)$ be sufficiently PE and $b$ be "relatively small."

Note that (6) is of the form (8), with $x=e_{1}, a(t):=$ $k_{2} y_{s}(t)^{2}$, and $b=\beta$. From Lemma 1 , it readily follows that, if $y_{s}$ is PE (which holds if the slave system has a chaotic behavior), $k_{2}$ is sufficiently large, and $\beta$ is "small," the origin of (6) is globally exponentially stable.

Step 2) The previous arguments rely on the assumption that $e_{3}=k_{2} y_{s}(t) e_{1}$. Hence, a controller that asymptotically makes $e_{3} \rightarrow k_{2} y_{s}(t) e_{1}$ must be designed under

\footnotetext{
${ }^{3}$ If, in addition, $\varphi$ is bounded and globally Lipschitz.
} 
the restriction of using only one control input. Consider (first) $u_{2} \equiv 0$ and

$$
u_{3}=-k e_{3}+k_{2} y_{s}(t) e_{1}, \quad k, k_{2}>0
$$

The choice of $u_{3}$ is motivated by the observation that, with $e_{2} \equiv e_{1} \equiv 0$, the $e_{3}$ dynamics has the form

$$
e_{3}(t)=\frac{1}{p+[k+b]} k_{2} y_{s}(t) e_{1}(t) \quad p:=\frac{d}{d t}
$$

i.e., the $e_{3}$ dynamics may be regarded as a low-pass filter with cutting frequency at $k+b$. In other words, for sufficiently large $k$, the "input" $k_{2} y_{s}(t) e_{1}(t)$ is recovered at the filter's output, up to a gain.

Step 3) In summary, the closed-loop system is

$$
\begin{aligned}
& {\left[\begin{array}{l}
\dot{e}_{1} \\
\dot{e}_{2}
\end{array}\right]=\left[\begin{array}{cc}
-\left[k_{2} y_{s}^{2}-\beta\right] & -z_{m} \\
z_{m} & -a
\end{array}\right]\left[\begin{array}{l}
e_{1} \\
e_{2}
\end{array}\right]+\left[\begin{array}{c}
-y_{s} \\
x_{s}
\end{array}\right] e_{3}} \\
& e_{3}(t)=\frac{1}{p+[k+b]} k_{2} y_{s}(t) e_{1}(t)+\left[\begin{array}{ll}
y_{m} & x_{s}
\end{array}\right]\left[\begin{array}{l}
e_{1} \\
e_{2}
\end{array}\right] .
\end{aligned}
$$

Roughly speaking, the system in (12a) is seen as a stable system with input $e_{3}$, whereas the "filter" system (12b) is also a stable system with additive "perturbation" $\left[\begin{array}{ll}e_{1} & e_{2}\end{array}\right]^{\top}$. A smallgain argument (cf. [3]) suffices to ensure the stability of the interconnected system; roughly, it is required that the respective terms multiplying the inputs are not too "large" relative to the stabilizing terms of the "nominal" systems. These intuitive arguments are recovered in Proposition 1; the proof is presented in Section III.

Proposition 1: Consider system (5) in closed loop with $u_{2} \equiv$ 0 and $u_{3}$, as in (10). Then, for sufficiently large gains $k$ and $k_{2}$, the origin of the closed-loop system is globally exponentially stable if $y_{s}(t)$ is PE with sufficiently large $\mu_{m}$ and $\Delta$.

Following the same train of thought that leads to Proposition 1, let us now explore the case when $u_{3} \equiv 0$ and use $u_{2}$ as control input. In this case, $e_{2}$ in (5a) is seen as the virtual control input: If $e_{3}=0$ and $e_{2}=k_{4} z_{m} e_{1}$, with $k_{4}>0$, (5a) reads

$$
\dot{e}_{1}=-\left[k_{4} z_{m}(t)^{2}-\beta\right] e_{1}
$$

which is of the form (8); hence, by Lemma 1, the origin $e_{1}=0$ of (13) is exponentially stable for an appropriate choice of $k_{4}$ and PE $z_{m}(t)$ with sufficiently large $\mu_{m}$ and $\Delta$. With this in mind, define

$$
u_{2}=-k_{4} e_{2}-2 x_{s} e_{3}+k_{2} z_{m}(t) e_{1}, \quad k_{2}, k_{4}>0 .
$$

Thus, the closed-loop equation of (5b) with (13) becomes

$$
\dot{e}_{2}=-\left[a+k_{4}\right] e_{2}+\left[k_{2}+1\right] z_{m}(t) e_{1}-x_{s} e_{3} .
$$

Disregarding the term $-x_{s} e_{3},(15)$ may be rewritten as a lowpass filter with input $\left[k_{2}+1\right] z_{m} e_{1}$ and cutting frequency at $a+$ $k_{4}$, which may be set at will, i.e.,

$$
e_{2}(t)=\frac{1}{p+\left[a+k_{4}\right]}\left[k_{2}+1\right] z_{m}(t) e_{1}(t), \quad p:=\frac{d}{d t} .
$$

Proposition 2: Consider system (5) in closed loop with $u_{3} \equiv$ 0 and $u_{2}$, as in (14). Then, for sufficiently large $k_{2}$ and $k_{4}$, the origin of the closed-loop system is globally exponentially stable if $z_{m}(t)$ is PE with sufficiently large $\mu_{m}$ and $\Delta$.

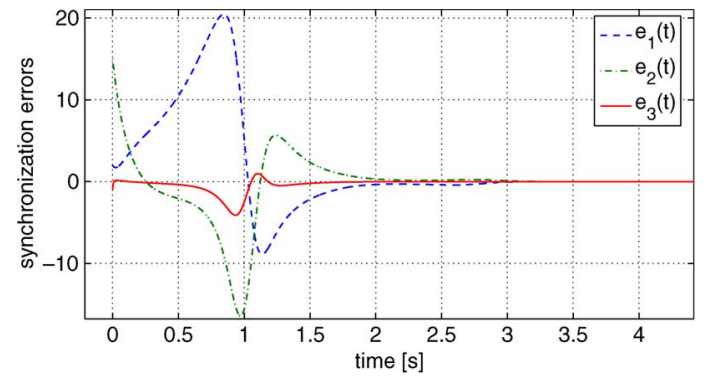

Fig. 2. Graphs of error trajectories $e(t)$ using control $u_{3}$ given by (10).

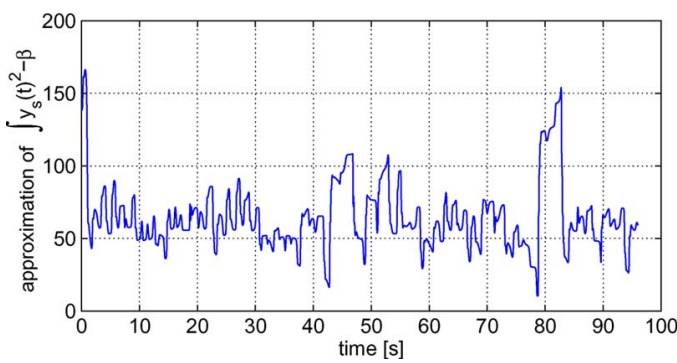

Fig. 3. Graph of the numerical approximation of $F(t):=\int_{t}^{t+\Delta}\left[y_{s}(s)^{2}-\right.$ $\beta] d s$ for $t \in[0,100-\Delta]$ under control $u_{3} ; \Delta=4 s$ and $\hat{\mu}_{m}:=$ $\min _{t \in[0,100-\Delta]}\{F(t)\}=10.4393$.

It is worth emphasizing that controller $u_{2}$ uses full master state feedback, whereas controller $u_{3}$ uses partial-state or output feedback (measurement of $z_{m}$ and $x_{m}$ only).

\section{Simulations}

We have tested the performance of controllers (10) and (14) in simulations using SIMULINK or MATLAB. The simulation is run for $100 \mathrm{~s}$ using a third-order approximation algorithm and an integration step of $1 e^{-4}$. The initial conditions are

$$
\begin{array}{rll}
x_{s}(0)=5 & y_{s}(0)=-5 & z_{s}(0)=1 \\
x_{m}(0)=3 & y_{m}(0)=-20 & z_{m}(0)=2
\end{array}
$$

and the parameters are selected, so that the systems describe a chaotic behavior: $a=-10, b=-4, c=0$, and $\beta=2.8571$. For the controller (10), the gains are set to $k=200$ and $k_{2}=1$. The results are shown in Figs. 2 and 3.

In the former, the synchronization errors exponentially tending to zero are shown; in the latter, the numerical integration of $\left[y_{s}(s)^{2}-\beta\right]$ is depicted. Note that the PE condition may be verified only numerically since it must be verified for all $t \geq 0$ : Let $\left\{\bar{y}_{s}\left(t_{j}\right)\right\}$ be an ordered array of points such that $\bar{y}_{s}\left(t_{j}\right)=y(t)$ for all $t \in[0,100]$ such that $t=t_{j} \in\left\{0,1 e^{-4}, 2 e^{-4}, \ldots, 100\right\}$, i.e., $\left\{\bar{y}_{s}\left(t_{j}\right)\right\}$ is the array of points generated by the numerical integration routine with a step of $0.1 \mathrm{~ms}$. To lighten the computational burden, let

$$
F(t):=\int_{t}^{t+4}\left[y_{s}(\tau)^{2}-\beta\right] d \tau \quad \forall t \in[0,100]
$$

by $F\left(t_{k}\right)$, where $\left\{t_{k}\right\}:=\{0,0.1,0.2, \ldots, 96\}$

$$
\begin{aligned}
F\left(t_{k}\right):=\sum_{t_{j}=10^{4} t_{k}}^{\left(t_{k}+4\right) 10^{4}}\left[\bar{y}_{s}\left(t_{j}\right)^{2}-\beta\right], \\
k \in\{1,2, \ldots, 9600\} ; \quad t_{j} \in \mathbb{Z}
\end{aligned}
$$




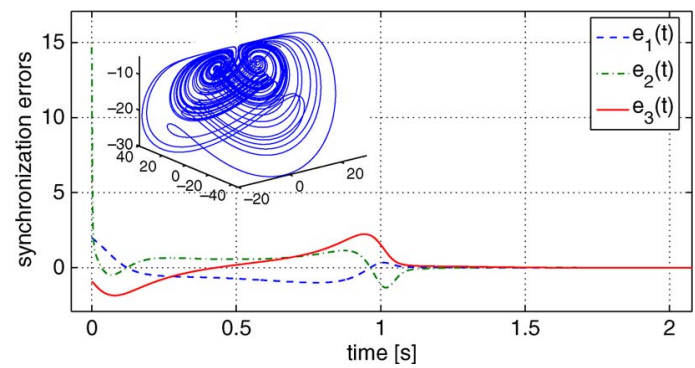

Fig. 4. Graphs of error trajectories $e(t)$ using control $u_{2}$ given by (14).

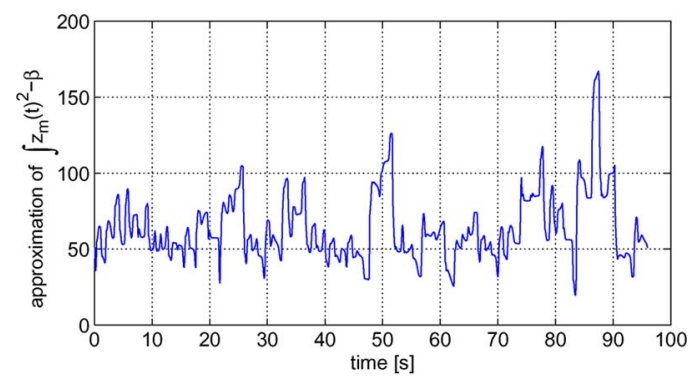

Fig. 5. Graph of the numerical approximation of $F(t):=\int_{t}^{t+\Delta}\left[z_{m}(s)^{2}-\right.$ $\beta] d s$ for $t \in[0,100-\Delta]$ under control $u_{2} ; \Delta=4 s$ and $\hat{\mu}_{m}:=$ $\min _{t \in[0,100-\Delta]}\{F(t)\}=19.60618$.

i.e., define a subsequence of 9600 points $\left\{t_{k}\right\}$ from the sequence $\left\{t_{j}\right\}, j \in \mathbb{Z}_{>0}, j \leq 10^{6}$, generated via the integration routine. This comes to computing 9600 sums of $4 \times 10^{4}$ terms each, as opposed to $1 e^{6}$ at the expense of resolution but not of accuracy. The minimal value of $F\left(t_{k}\right)$ gives an estimate of the PE bound $\mu_{m}$; in this particular example, it is $\hat{\mu}_{m}=10.439$ (see Fig. 3).

In a second run of simulations, the controller $u_{2}$ defined in (14) was tested under similar conditions. The results are shown in Figs. 4 and 5. As for the previous case, error trajectories $e_{i}(t)$ (cf. Fig. 4) and the graph of $F\left(t_{k}\right)$, defined in (17) albeit replacing $y_{s}$ with $z_{m}$, are shown. In Fig. 4 , the 3-D attractor for the slave system is shown.

Note that, in both Figs. 2 and 4, the short transients are comparable to those under nonlinear cancellation control with two inputs (cf. [11]) and with three inputs (cf. [10]).

\section{PROOFS}

Proof of Lemma 1: Let $\varphi_{M}>0$ be such that $|\varphi(t)| \leq \varphi_{M}$ for all $t$, and define

$V_{1}(x)=\left(\frac{1}{2}+\varphi_{M}\right) x^{2} \quad V_{2}(t, x):=-\int_{t}^{\infty} \varphi(\tau) x^{2} e^{t-\tau} d \tau$.

(Note that $V_{2}$ is reminiscent of a convolution integral.) Hence

$$
V_{2}(t, x) \leq-\int_{t}^{t+\Delta} \varphi(\tau) x^{2} e^{\Delta} d \tau \leq-\mu_{m} e^{\Delta} x^{2} .
$$

We also remark that

$$
\int_{t}^{\infty} \varphi(\tau) e^{t-\tau} d \tau \leq \varphi_{M} \int_{t}^{\infty} e^{t-\tau} d \tau=\varphi_{M}
$$

Thus, $V_{2} \geq-\varphi_{M} x^{2}$, and $V_{1}+V_{2}$ is positive definite. Now, evaluating the total time derivative of $V_{1}$ along the trajectories of (8) yields $\dot{V}_{1}=-\left[2 \varphi_{M}+1\right] \varphi(t) x^{2}$, whereas the time derivative of $V_{2}$ satisfies

$$
\dot{V}_{2}=\varphi(t) x^{2}+\int_{t}^{\infty} 2 x^{2} \varphi(\tau) \varphi(t) e^{t-\tau} d \tau+V_{2} .
$$

Using $|\varphi(t)| \leq \varphi_{M}$, it is concluded that the integral term in (19) is bounded by $2\left|V_{2}\right| \varphi_{M}$, so $\dot{V}_{1}+\dot{V}_{2} \leq-2 \varphi_{M} \varphi(t) x^{2}+$ $2\left|V_{2}\right| \varphi_{M}-\mu_{m} e^{\Delta} x^{2}$; therefore, using $\left|V_{2}\right| \leq \varphi_{M} x^{2}$ yields

$$
\dot{V}_{1}+\dot{V}_{2} \leq-\left[\mu_{m} e^{\Delta}-4 \varphi_{M}^{2}\right] x^{2}
$$

which is negative definite if $\mu_{m} e \Delta \geq 4 \varphi_{M}^{2}$, which holds for sufficiently large $\mu_{m}$ and $\Delta$. (Note, however, that this is a conservative bound.) Lastly, it is verified that, on the one hand, $|\varphi(\tau)| \leq \varphi_{M}$ and, on the other hand, (9) must hold by assumption. Hence, it is required that

$$
\mu_{m} \leq \int_{t}^{t+\Delta} \varphi(\tau) d \tau \leq \varphi_{M} \Delta
$$

which holds only if $\mu_{m} \leq \varphi_{M} \Delta$. The latter holds for sufficiently large $\Delta$.

Proof of Proposition 1: Define $e_{3 d}:=k_{2} y_{s} e_{1}$ and $\tilde{e}_{3}:=$ $e_{3}-e_{3 d}$. Then, the closed-loop equations (5), with $u_{1}=u_{2}=$ 0 , and (10) are

$$
\begin{aligned}
& \dot{e}_{1}=-\left[k_{2} y_{s}(t)^{2}-\beta\right] e_{1}-z_{m} e_{2}-y_{s} \tilde{e}_{3} \\
& \dot{e}_{2}=-a e_{2}+z_{m} e_{1}+x_{s} e_{3} \\
& \dot{e}_{3}=-[k+b] e_{3}+x_{s} e_{2}+\left[y_{m}+k_{2} y_{s}\right] e_{1} .
\end{aligned}
$$

Next, a dynamic equation for $\tilde{e}_{3}$ is derived, i.e.,

$$
\begin{aligned}
\dot{\tilde{e}}_{3}= & -(k+b)\left(\tilde{e}_{3}+e_{3 d}\right)+\left[y_{m} x_{s}\right]\left[\begin{array}{l}
e_{1} \\
e_{2}
\end{array}\right]+k_{2} y_{s} e_{1} \\
& -k_{2} \dot{y}_{s} e_{1}-k_{2} y_{s}\left[-\left(k_{2} y_{s}^{2}-\beta\right) e_{1}-z_{m} e_{2}-y_{s} \tilde{e}_{3}\right] \\
= & -[k+b] \tilde{e}_{3}-[k+b] e_{3 d}+y_{m} e_{1}+x_{s} e_{2}+k_{2} y_{s} e_{1} \\
& -k_{2} \dot{y}_{s} e_{1}+k_{2} y_{s}\left[k_{2} y_{s}^{2}-\beta\right] e_{1}+k_{2} z_{m} y_{s} e_{2}+k_{2} y_{s}^{2} \tilde{e}_{3} .
\end{aligned}
$$

Using $e_{3 d}=k_{2} y_{s} e_{1}$ and regrouping terms yield

$$
\begin{aligned}
\dot{\tilde{e}}_{3}=-[k+b] \tilde{e}_{3}+ & e_{1}\left[y_{m}+k_{2} y_{s}-k_{2} \dot{y}_{s}-k_{2}(k+b) y_{s}\right] \\
& +e_{3 d}\left[k_{2} y_{s}^{2}-\beta\right]+e_{2}\left[x_{s}+z_{m} y_{s}\right]+y_{s}^{2} \tilde{e}_{3} .
\end{aligned}
$$

Using $e_{3 d}=e_{3}-\tilde{e}_{3}$ results in

$$
\begin{aligned}
\dot{\tilde{e}}_{3}=-[k+b & -\beta] \tilde{e}_{3}+e_{3}\left[k_{2} y_{s}^{2}-\beta\right]+e_{2}\left[x_{s}+y_{s} z_{m}\right] \\
& +e_{1}\left[y_{m}-k_{2}(k+b) y_{s}+k_{2} y_{s}-k_{2} \dot{y}_{s}\right] .
\end{aligned}
$$

Define $\Psi(t)^{\top}:=\left[\psi_{1}(t) \psi_{2}(t) \psi_{3}(t)\right]$, with $\psi_{1}(t):=y_{m}(t)+$ $k_{2}\left[y_{s}(1-k+b)-\dot{y}_{s}\right], \psi_{2}(t):=x_{s}(t)+y_{s}(t) z_{m}(t)$, and $\psi_{3}(t):=$ $k_{2} y_{s}(t)^{2}-\beta$. Then

$$
\dot{\tilde{e}}_{3}=-(k+b-\beta) \tilde{e}_{3}+\Psi(t)^{\top} e, \quad e^{\top}:=\left[e_{1} e_{2} e_{3}\right] .
$$

Let $e_{12}^{\top}:=\left[\begin{array}{ll}e_{1} & e_{2}\end{array}\right]$ and $V_{12}\left(t, e_{12}\right):=V_{1}\left(e_{1}\right)+V_{2}\left(t, e_{1}\right)+$ $0.5 e_{2}^{2}$, where $V_{1}$ and $V_{2}$ are defined in (18) and $V_{3}:=0.5 e_{3}^{2}$. Following the proof of Lemma 1 leads to

$$
\begin{aligned}
\dot{V}_{12}+\dot{V}_{3} \leq & -\left[\mu_{m} e^{\Delta}-4 \varphi_{M}^{2}\right] e_{1}^{2}-a e_{2}^{2}-[k+b] e_{3}^{2}+2 e_{2} e_{3} x_{s} \\
& +\left[y_{m}+k_{2} y_{s}\right] e_{1} e_{3}+\left[4 \varphi_{M}+1\right]\left|y_{s}\right|\left|\tilde{e}_{3}\right|\left|e_{1}\right| \\
& +4 \varphi_{M}\left|e_{1}\right|\left|e_{2}\right|\left|z_{m}\right|
\end{aligned}
$$


where the equality

$$
\frac{\partial V_{12}}{\partial e_{1}}=\left[2 \varphi_{M}+1-2 \int_{t}^{\infty} \varphi(\tau) e^{t-\tau} d \tau\right] e_{1} \leq\left[4 \varphi_{M}+1\right]\left|e_{1}\right|
$$

was used. Define $\tilde{V}_{3}:=0.5 \tilde{e}_{3}^{2}$. Then,

$$
\dot{\tilde{V}}_{3}=-[k+b-\beta] \tilde{e}_{3}^{2}+\Psi(t)^{\top} e \tilde{e}_{3} .
$$

Finally, define $\kappa:=\left[4 \varphi_{M}+1\right]\left|y_{s}\right|$ and $\mathcal{V}:=\gamma V_{12}+V_{3}+$ $\tilde{V}_{3}$, with $\gamma \propto(k+b)$; its total time derivative satisfies

$$
\begin{aligned}
2 \dot{\mathcal{V}} & \leq\left[\begin{array}{ll}
e^{\top} & \tilde{e}_{3}
\end{array}\right]^{\top} D\left[\begin{array}{c}
e \\
\tilde{e}_{3}
\end{array}\right]-\gamma\left[\mu_{m} e^{\Delta}-4 \varphi_{M}^{2}\right] e_{1}^{2}-a \gamma e_{2}^{2}-[k+b] e_{3}^{2} \\
D & :=\left[\begin{array}{cccc}
-\gamma\left(\mu_{m} e^{\Delta}-4 \varphi_{M}^{2}\right) & 4 \gamma \varphi_{M}\left|z_{m}\right| & \left(y_{m}+k_{2} y_{s}\right) & \gamma \kappa+\psi_{1} \\
4 \gamma \varphi_{M}\left|z_{m}\right| & -a \gamma & x_{s} & \psi_{2} \\
\left(y_{m}+k_{2} y_{s}\right) & x_{s} & -[k+b] & \psi_{3} \\
\gamma \kappa+\psi_{1} & \psi_{2} & \psi_{3} & -[k+b-\beta]
\end{array}\right] .
\end{aligned}
$$

Since the off-diagonal elements of matrix $D$ are bounded, ${ }^{4}$ $D$ may be made negative definite for sufficiently large values of design parameters $\mu_{m}, \Delta, k$, and $k_{2}$, so $\dot{\mathcal{V}}$ is negative definite.

Proof of Proposition 2: The proof follows similar guidelines. In this case, with $\tilde{e}_{2}=e_{2}-e_{2 d}$ and $e_{2 d}=k_{2} z_{m} e_{1}$, the closedloop equations (5) with (14) and $u_{3}=0$ yield

$$
\begin{aligned}
& \dot{e}_{1}=-\left[k_{2} z_{m}(t)^{2}-\beta\right] e_{1}-z_{m} \tilde{e}_{2}-y_{s} e_{3} \\
& \dot{e}_{2}=-\left[k_{4}+a\right] e_{2}+z_{m}\left(k_{2}+1\right) e_{1}-x_{s} e_{3} \\
& \dot{e}_{3}=-b e_{3}+x_{s} e_{2}+e_{1} y_{m} .
\end{aligned}
$$

Following somewhat lengthy but direct computations, as to obtain $\dot{\tilde{e}}_{3}$ in the proof of Proposition 1 leads to

$$
\begin{array}{r}
\dot{\tilde{e}}_{2}=-\left[k_{4}+a-\beta\right] \tilde{e}_{2}-\left[x_{s}-k_{2} z_{m} y_{s}\right] e_{3}+\left(k_{2} z_{m}^{2}-\beta\right) e_{2} \\
-\left[\left(k_{4}+a\right) k_{2} z_{m}-\left(k_{2}+1\right) z_{m}+\dot{z}_{m}\right] e_{1} .
\end{array}
$$

Define $e_{12}^{\top}:=\left[\begin{array}{ll}e_{1} & e_{2}\end{array}\right]$, and consider function $V_{12}\left(t, e_{1}\right):=$ $V_{1}\left(e_{1}\right)+V_{2}\left(t, e_{1}\right)$. Let $z_{m}$ be (sufficiently) PE, so that $\left(k_{2} z_{m}^{2}-\beta\right)$ is also $\mathrm{PE}^{5}$ with $\mu_{m}$ and $\Delta$. The total time derivative using (23a) satisfies

$$
\dot{V}_{12} \leq-\left[\mu_{m} e^{\Delta}-4 \varphi_{m}^{2}\right] e_{1}^{2}+\left[6 \varphi_{m}+1\right]\left(\left|e_{3} y_{s}\right|+\left|z_{m} \tilde{e}_{2}\right|\right)\left|e_{1}\right| \text {. }
$$

Next, define $V_{23}\left(e_{23}\right):=0.5\left|e_{23}\right|^{2}$. We have

$$
\dot{V}\left(e_{23}\right) \leq-\left(k_{4}+a\right) e_{2}^{2}-b e_{3}^{2}+\left|e_{1}\right|\left(\left|z_{m}\right|\left|e_{2}\right|+\left|y_{m}\right|\left|e_{3}\right|\right) \text {. }
$$

Collecting terms yields

$$
\begin{gathered}
\dot{V}_{12}+\dot{V}_{23} \leq \frac{1}{2}\left[\begin{array}{l}
\left|e_{1}\right| \\
\left|e_{2}\right| \\
\left|e_{3}\right|
\end{array}\right]^{\top} M_{\lambda}\left[\begin{array}{l}
\left|e_{1}\right| \\
\left|e_{2}\right| \\
\left|e_{3}\right|
\end{array}\right]+\left(6 \varphi_{m}+1\right)\left|z_{m}\right|\left|e_{1}\right|\left|\tilde{e}_{2}\right| \\
M_{\lambda}:=\left[\begin{array}{ccc}
-\lambda\left(\mu_{m} e^{\top}-4 \varphi_{m}^{2}\right) & \left|z_{m}\right| & \left|y_{m}\right|+\left|y_{s}\right|\left(6 \varphi_{m}+1\right) \\
\left|z_{m}\right| & -\left(k_{4}+a\right) & 0 \\
\left|y_{m}\right|+\left|y_{s}\right|\left(6 \varphi_{m}+1\right) & 0 & -b
\end{array}\right]
\end{gathered}
$$

with $\lambda \in(0,1]$. Matrix $M_{\lambda}$ is negative definite if

$$
\lambda\left(\mu_{m} e^{\Delta}-4 \varphi_{M}^{2}\right)\left(k_{4}+a\right) b>\left(k_{4}+a\right)\left(6 \varphi_{M}+1\right)^{2}+z_{m}^{2} y_{m}^{2} b
$$

\footnotetext{
${ }^{4}$ This may be shown under the assumption that the slave system operates in the chaotic regime, in open loop.

${ }^{5}$ Correspondingly, it is required that $\beta$ is relatively small, i.e., that a PE signal remains $\mathrm{PE}$ under the addition of a small constant is a well-known property of PE functions.
}

which holds for sufficiently large $\mu_{m}$ and $\Delta$ for any fixed $\lambda$. Let $\lambda \in(0,1), \lambda \approx 1, c:=\lambda_{\min }\left(M_{\lambda}\right)$ (the smallest eigenvalue of $\left.M_{\lambda}\right)$ and $\gamma>0$. Define $\mathcal{V}(e, \tilde{e}):=V_{12}\left(e_{1}\right)+V_{23}\left(e_{23}\right)+$ $0.5 \gamma \tilde{e}_{2}^{2}$ and $\Psi:=\left[\begin{array}{lll}\psi_{1} & \psi_{2} & \psi_{3}\end{array}\right]^{\top}$, with $\psi_{1}:=-\left[\left(k_{4}+a\right) k_{2} z_{m}-\right.$ $\left.z_{m}+\dot{z}_{m}\right], \psi_{2}:=\left(k_{2} z_{m}^{2}-\beta\right)$, and $\psi_{3}:=-\left[x_{s}-k_{2} z_{m} y_{s}\right]$. Then

$$
\begin{aligned}
2 \dot{\mathcal{V}} \leq & -c|e|^{2}-\left[k_{4}+a-\beta\right] \gamma \tilde{e}_{2}^{2} \\
& -\left[\begin{array}{c}
|e| \\
\tilde{e}_{2}
\end{array}\right]^{\top}\left[\begin{array}{cc}
c & \gamma|\Psi| \\
\gamma|\Psi| & \frac{1}{2} \gamma\left[k_{4}+a-\beta\right]
\end{array}\right]\left[\begin{array}{c}
|e| \\
\tilde{e}_{2}
\end{array}\right] \\
& -\left[\begin{array}{c}
e_{1} \\
\tilde{e}_{2}
\end{array}\right]^{\top}\left[\begin{array}{cc}
(1-\lambda)\left(\mu_{m} e^{\Delta}-4 \varphi_{m}^{2}\right) & \left(6 \varphi_{m}+1\right)\left|z_{m}\right| \\
\left(6 \varphi_{m}+1\right)\left|z_{m}\right| & \frac{1}{2} \gamma\left[k_{4}+a-\beta\right]
\end{array}\right] \\
& \times\left[\begin{array}{c}
e_{1} \\
\tilde{e}_{2}
\end{array}\right] .
\end{aligned}
$$

The last two terms on the right-hand side of the preceding expression are nonpositive if

$$
\begin{aligned}
& {\left[k_{4}+a-\beta\right] c \geq 2 \gamma|\Psi|^{2}} \\
& {\left[k_{4}+a-\beta\right] \gamma(1-\lambda)\left(\mu_{m} e^{\Delta}-4 \varphi_{m}^{2}\right) \geq\left(6 \varphi_{m}+1\right)^{2} z_{m}^{2} .}
\end{aligned}
$$

Both inequalities are satisfied for sufficiently small $\gamma$ and sufficiently large $k_{4}, a, \mu_{m}$, and $\Delta$.

We conclude that $\mathcal{V}$ is negative definite. Moreover, since $\mathcal{V}$ satisfies lower and upper quadratic bounds of the norm of $\left[e \tilde{e}_{2}\right]$, global exponential stability follows.

$\Delta \Delta$

\section{CONCLUSION}

We have shown that master-slave synchronization of Lü chaotic oscillators is achievable with one control input. Moreover, our control laws are linear time varying. Further research is targeted at solving the problem under parameter uncertainty, i.e., using adaptive control.

\section{REFERENCES}

[1] B. O. Anderson, "Exponential stability of linear equations arising in adaptive identification," IEEE Trans. Autom. Control, vol. AC-22, no. 1, pp. 83-88, Feb. 1977.

[2] E. Cherrier, M. Boutayeb, and J. Ragot, "Observers-based synchronization and input recovery for a class of nonlinear chaotic models," IEEE Trans. Circuits Syst. I, Reg. Papers, vol. 53, no. 9, pp. 1977-1988, Sep. 2006.

[3] G. Escobar, M. Hernández-Gómez, and P. R. Martínez-Montejano, "A repetitive-based controller for a power factor precompensator," IEEE Trans. Circuits Syst. I, Reg. Papers, vol. 54, no. 9, pp. 1968-1976, Sep. 2007.

[4] M. L'Hernault, J.-P. Barbot, and A. Ouslimani, "Feasibility of analog realization of a sliding-mode observer: Application to data transmission," IEEE Trans. Circuits Syst. I, Reg. Papers, vol. 55, no. 2, pp. 614-624, Mar. 2008.

[5] G.-P. Jiang, W. X. Zheng, W. K.-S. Tang, and G. Chen, "Integral-observerbased chaos synchronization," IEEE Trans. Circuits Syst. II, Exp. Briefs, vol. 53, no. 2, pp. 110-114, Feb. 2006.

[6] K. Kemih and M. Benslama, "Synchronized chaotic Lü system in CDMA satellite communication using genetic algorithm," in Proc. ICDT, 2006, pp. 33-37.

[7] H. Khalil, Nonlinear Systems, 3rd ed. New York: Prentice-Hall, 2002.

[8] A. Loría and A. Zavala, "Adaptive tracking control of chaotic systems with applications to synchronization," IEEE Trans. Circuits Syst. I, Reg. Papers, vol. 54, no. 9, pp. 2019-2029, Sep. 2007.

[9] J. Lü, G. Chen, and D. Cheng, "A new chaotic system and beyond: The generalized Lorenz-like system," Int. J. Bifurc. Chaos, vol. 14, no. 5, pp. 1507-1537, May 2004.

[10] J. H. Park, "Chaos synchronization of a chaotic system via nonlinear control," Chaos Solitons Fractals, vol. 25, no. 3, pp. 579-584, Aug. 2005.

[11] Q. Zhang and J. Lu, "Chaos synchronization of a new chaotic system via nonlinear control," Chaos Solitons Fractals, vol. 37, no. 1, pp. 175-179, Jul. 2008. 